\title{
Improving accuracy of intention-based response classification using decision tree.
}

\begin{abstract}
This study focused on improving the dialogue act classification to classify a user utterance into a response class using a decision tree approach. Decision tree classifier is tested on 64 mixed-initiative, transaction dialogue corpus in theater domain. The result from the comparative experiment show that decision tree able to achieve $81.95 \%$ recognition accuracy in classification better than the $73.9 \%$ obtained using Bayesian networks and $71.3 \%$ achieved by using Maximum likelihood estimation. This result showed that the performance of decision tree as classifier is well suited for these tasks.
\end{abstract}

Keyword: Classification; Decision tree; Natural language generation; Dialogue systems. 